\title{
Model and Empirical Research on Private Equity Investment
}

\section{Performance}

\author{
Bin Zhu ${ }^{1, a}$, Yuanyuan Wang ${ }^{2, b,{ }^{*}}$ and Yixiao Meng ${ }^{3, c}$ \\ 1,2,3 School of Management, Harbin Institute of Technology, Harbin,Heilongjiang,China \\ azb@hit.edu.cn, bhit10wyy@163.com, 'lyre038@163.com \\ ${ }^{*}$ Corresponding author
}

\begin{abstract}
As a modern investment and financing methods, whether for investors or fund managers, the good performance of private equity is very important. This paper choses empirical research methods, private equity funds exiting cases between 2003-2013for the data base, the Public Market Equivalent (PME) as a representative of the private equity performance indicators. Regression model was constructed to study the effect to the PME by the amount of investment, the investment of time and the internal rate of return. Descriptive model was constructed to study the effect to the investment return and book return by the exit way and the industry type.
\end{abstract}

Keywords: private equity; investment performance; public market equivalent

\section{私募股权投资绩效模型与实证研究}

\author{
朱涁 ${ }^{1, a}$, 王媛媛 ${ }^{2, b,{ }^{*}}$, 孟义晓 ${ }^{3, c}$ \\ $1,2,3$ 哈尔滨工业大学管理学院, 哈尔滨, 黑龙江省, 中国 \\ azb@hit.edu.cn, bhit10wyy@163.com, ${ }^{\mathrm{C}} l y r e 038 @ 163 . c o m$
}

中文摘要: 私募股权投资作为现代投资和融 资方式, 不论对投资者还是对资金管理者来 说良好的绩效都是其目的所在。本文以 2003 年至 2013 年十年间私募通数据库所收 录的私募股权基金退出案例为数据基础, 选 用实证研究方法, 将公共市场等价（PME） 作为私募股权绩效的代表性指标, 分别构建 了回归模型与描述性模型，研究投资金额、 投资时间、内部收益率对公共市场等价的影 响, 退出方式、行业类型对私募股权投资回 报率及账面回报的影响, 不同年份的公共市 场等价分析。

关键词: 私募股权; 投资绩效; 公共市场等 价

\section{一、引言}

私募股权投资作为现代投资和融资方 式的一种, 不论对投资者还是对资金管理者 来说良好的绩效都是其目的所在。尤其是对 于资金的管理者来说, 基金的绩效不仅影响 其自身的收益, 还决定了其未来进一步募集 资金的能力。为了对我国的私募股权基金的 运营效果即其绩效水平进行考量, 考察其具 体的收益水平,有必要对我国的私募股权基 金的市场绩效进行研究。

近几十年, 国内外学者对私募股权绩效 及影响因素等方面做了诸多研究。Brav 和 Gompers(1997)通过对比由风险投资支持的 
IPO 和没有风险投资支持的 IPO 公司的长 期绩效表现指出由风险投资支持的 IPO 要 优于没有风险投资支持的 IPO。

Cochrane(2004)使用 Venture One 的数据基 于投资公司层面对私募股权基金的绩效进 行了分析, 在对结果进行生存偏差调整后, 作者指出平均的收益水平为 59\%, 标准差 水平为 $107 \%$ 。赖继红(2012) 应用我国创业 板上市公司经验数据分两阶段检验了私募 股权投资与企业创新, 指出在理论上, 企业 家与私募股权投资均会通过促进创新来促 进企业增长, 但经验数据却反映出我国私募 股权投资对企业的创新影响微弱。Diller 和 Kaserer(2009) 基于私募股权基金流动性 差, 粘性和分割的特征指出, Gompers 和 Lerner(2000) 提出的 “金钱追逐交易” 的现 象解释了私募股权基金收益中的很大一部 分。并指出市场敏感性, 普通合伙人的技能 以及单独的投资风险都对私募股权基金的 绩效有影响。郑超(2011) 对私募股权投资 项目层次的收益进行了实证分析, 指出投资 时间、退出方式、退出市场对项目账面退出 回报倍数存在显著的影响。投资时间对项目 退出回报具有正向影响, 但与账面内部收益 率具有反向关系。赎尔翔(2012) 等从联合 投资的角度分析了私募股权基金的绩效, 并 指出中国市场的数据表明投资网络对于领 投者和跟投者的投资绩效的确能够带来正 向影响, 但如果投资网络为结构洞信息流交 换机制, 则有可能因各种道德问题而降低整 个投资联盟的投资绩效, 且私募股权投资者 受到投资网络的限制越多, 其投资绩效越 低。

综上所述, 国外目前关于私募股权基金 绩效的研究比国内要更加深入, 无论是在研 究的范围上还是在研究的方法上都比国内 更加细淢。国内目前关于私募股权投资绩效 的研究虽然也有很大的发展, 但受制于数据 获取等原因目前的研究并不深入。一方面, 表现为研究的对象不够丰富, 虽然现在对私 募股权基金绩效的研究较多, 但大多集中在 产业（企业）层面，对基金自身收益问题的 研究相对较少。另一方面, 研究的方法不够 深入, 国外对私募股权投资绩效的研究多采 用实证的方法, 通过统计分析指出私募股权
基金收益与公共市场绩效的差异,而国内对 私募股权基金绩效的分析则多采用理论分 析的方法, 没有对私募股权基金在基金层面 进行深入的统计分析。本文通过搜集相关的 数据, 借鉴国内外现有研究, 对我国私募股 权投资的绩效在基金层面进行研究。

\section{二、私募股权投资绩效分析与指标}

本文采用广义的私募股权的概念, 即从 非公开渠道募集资金并进行投资,通过上市 或并购实现资金退出的投资，包括风险投 资、并购、夹层资本等传统意义上的私募投 资都称为私募股权投资。

\section{1 私募股权投资绩效分析}

对私募股权投资绩效的分析, 可以从总 体绩效水平、退出方式、行业影响以及公共 市场等价四个方面进行。

私募股权投资的总体绩效水平受到多种 因素的影响，投资时间长短不同，投资周期 内投资对象所处的生命周期即有所不同, 投 资的风险程度、收益增长速度均有所不同, 从而影响私募股权的总体绩效水平; 投资金 额大小会影响资金的规模效应, 也会对私募 股权的总体积小水平产生影响。私募股权投 资的退出方式主要包括 IPO、并购、管理层 回购与反向收购四种方式, 不同退出方式的 成交条件、成交周期、风险大小也有所不同， 因此不同的退出方式产生的投资绩效也各 不相同。不同行业的行业特征有着显著区 别, 由于私募股权投资对象多为初创企业, 行业准入门槛的高低、行业生命周期的分布 以及行业标准、盈利水平等因素, 均会对投 资行业的选择和投资的绩效表现产生很大 影响。公共市场等价 (PME) 可以衡量同等 数量的资金投资于非公开交易市场与投资 于公开交易市场两者获利水平。

\section{2 私募股权投资绩效指标}

在私募股权投资行业, 衡量其绩效的指 标通常有三个: 账面回报、内部收益率和公 共市场等价。在以上四个方面涉及到的绩效 表现分析中, 除了上述三个指标之外, 本文 还选取了投资时间、投资金额两个指标。

账面回报衡量的是一单位投资所产生 的收入, 即所产生的账面回报与投资的比 值。内部收益率指项目净现值为零时的折现 率。公共市场等价作为衡量非公开交易市场 
与公开交易市场差异的指标也将用于私募 股权投资绩效的分析。关于公共市场等价的 计算则是通过考察私募股权退出的回报收 益与同样的投资在相同的时间段内在公共 市场的收益相比较得出, 其理论基础是价格 市场等价理论。投资时间衡量的是私募股权 基金在将资金投入到企业后到企业上市或 被兼并时所经历的时间。投资金额即私募股 权基金投资到被投资企业中的资金量。

\section{三、私募股权投资绩效模型设计}

\section{1 绩效模型设计}

许多学者在研究中会使用回归模型对 自变量与因变量之间的关系进行研究, 在选 取自变量时往往即有连续变量也有分类变 量, 而分类变量如行业类型、退出方式等不 能直接进行回归, 需要对其进行虚拟化处理 才能进行线性回归, 在回归结果解释方面不 如连续变量直观易懂。因此考虑到分类变量 的特殊属性, 在进行私募股权投资绩效分析 时采用线性回归模型, 但并不把诸如行业类 型、退出方式等分类变量作为自变量进行回 归分析, 而是建立描述性分析模型, 对其单 独分析, 研究不同分类变量下的私募股权投 资绩效现状。

\subsection{1 回归模型}

在回归分析中, 选取公共市场等价作为 回归模型的因变量, 从而对引起公开交易市 场与非公开交易市场绩效差异的因素与两 个市场之间的绩效差异进行统计研究。分析 模型的表达式如下 (1):

$\mathrm{LN}\left(\mathrm{PME}{ }^{\prime}\right)=\mathrm{c}+\alpha * \mathrm{LN}($ investment $)+\beta * \mathrm{LN}($ time $)$

$$
+\gamma * \mathrm{LN}(\mathrm{irr})+\varepsilon
$$

式中, PME'一公共市场等价;

investment一投资金额;

time一投资时间;

irr一内部收益率;

$\varepsilon$ 一残差项。

将该模型用 PME'的 $n$ 个观测值和与之 相联系的投资金额、投资时间、内部收益率 的值来表示, 则模型可以表示为:

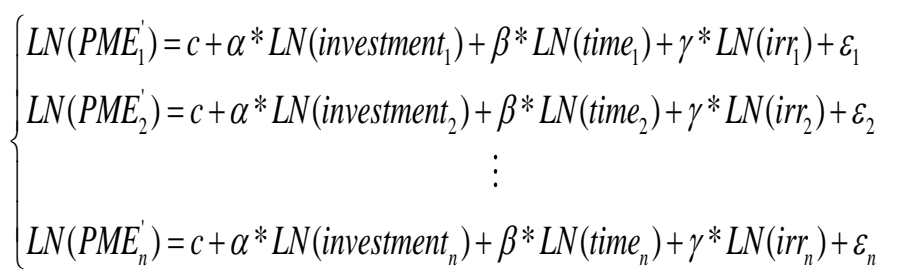

其中, 误差项假定具有以下性质:

(1) $\mathrm{E}\left(\varepsilon_{\mathrm{i}}\right)=0$;

(2) $\operatorname{Var}\left(\varepsilon_{\mathrm{i}}\right)=\sigma^{2}$ (常数);

(3) $\operatorname{Cov}\left(\varepsilon_{\mathrm{i}}, \varepsilon_{\mathrm{j}}\right)=0, \mathrm{i} \neq \mathrm{j}$.

2.1.2 描述性模型

在描述性分析中, 以内部收益率、账面 回报、投资金额、投资时间作为衡量绩效表 现的主要指标, 通过对其均值、标准差、离 差系数的统计分析, 对不同退出方式、行业 类型以及不同年份的公共市场等价 (PME) 进行了分析。

为衡量私募股权投资绩效的风险水平， 引入离差系数的概念。离差系数表明数据的 离散程度, 通常用来衡量风险水平。 $C_{\mathrm{v}}$ 值越 小则表示数据越集中, 风险也较小; $\mathrm{C}_{\mathrm{v}}$ 值越 大, 则表示数据越离散, 风险也较大。

\section{2 数据来源}

本文分析所选样本来自清科集团私募 通数据库。由于本文对私募股权的定义采取 广义上的理解, 即将私募股权投资与风险投 资统称为私募股权投资, 因此不需再对数据 库中的退出案例进行私募股权与风险投资 的划分, 而是将其整体作为分析的对象。本 文分析的数据以自 2003 年 3 月份起至 2013 年 12 月份止期间私募通数据库所收录的私 募股权基金退出案例为基础,剔除数据全无 的样本, 账面回报、内部收益率数据不完整 的样本, 以及有明显错误的样本 (账面回报 小于 1 的其内部收益率应为负值, 但在列表 中却为正值), 余下的案例即为本文分析的 样本, 共 1505 个案例样本。

\section{四、实证分析及结论}

\section{1 回归分析}

对公共市场等价等数据进行回归分析, 回归结果见表 $3-1 、 3-2$ 。 
表3-1 回归结果

\begin{tabular}{|c|c|c|c|c|}
\hline 模型 & $\mathbf{R}$ 值 & $\mathbf{R 2}$ & 调整 R2 & Sig \\
\hline 1 & 0.788 & 0.621 & 0.620 & 0.000 \\
\hline
\end{tabular}

表3-2 回归结果

\begin{tabular}{|c|c|c|}
\hline 变量 & 非标准系数 & $\mathrm{Sig}$ \\
\hline 常数 & 1.058 & 0.00 \\
\hline 投资金额 & -.057 & 0.00 \\
\hline 投资时间 & 1.031 & 0.00 \\
\hline 内部收益率 & .620 & 0.00 \\
\hline
\end{tabular}

根据表 3-1、3-2 结果显示模型的 $R^{2}$ 为 0.621 , 表明该模型可以解释自变量 $62.1 \%$ 的变差, 拟合效果较好。ANOVA 分析显示 Sig. 值为 0.000 , 小于 0.05 , 表明该回归模型在 显著性水平上是显著的。Coefficiens 分析 显示各自变量的 Sig. 值均小于 0.05, 因此 统计变量在显著性上也是显著的。

据此, 得出模型的最终结果为:

LN (PME' ) $=1.058-0.057 * \ln$ (invesmen )$+1.031 * \ln ($ time $)+0.620 * \ln ($ irr $)$

该模型表明投资金额与公共市场等价 的关系为负相关关系, 在其他变量不变的情 况下, 投资增加一单位, 公共市场等价将降 低 0.057 个单位; 而投资时间与内部收益率 与公共市场等价均为正相关关系, 投资时间 增加一单位, 公共市场等价增加 1.031 个单 位, 内部收益率增加一单位, 公共市场等价 增加 0.62 个单位。

\section{2 描述性统计分析}

\subsection{1 退出方式分析}

在对退出方式的统计中, IP0 退出共 1308 个案例, 并购退出 159 个案例, 管理 层回购退出 30 个案例, 反向收购退出 4 个 案例, 另有四个退出类型未知的案例, 不在 统计范围之内。由于反向收购只有四个案 例, 代表性不强, 因此并不作为统计分析的 基础。

由统计结果可知, IP0 退出方式是私募 股权投资者最青睐的退出方式, 占比 86. $91 \%$, 内部收益率均值为 1.41 (141\%), 账面回报均值为 8.52 倍, 均高于其他退出 方式, 且投资时间均值也是三种退出方式中 最小的。并购退出也是投资者较为青睐的退
出方式, 并购的账面回报离差系数 (1.65) 小于 IP0 的账面回报离差系数 (2.53), 且投 资时间离差系数 $(0.68)$ 也小于 IP0 的投资 时间离差系数 (0.73), 说明从账面回报和投 资时间风险的角度并购退出要优于 IP0 退 出回报。管理层回购相比这两种退出方式其 优势并不明显, 但从风险的角度考察绩效表 现, 管理层回购却有其优势, 其内部收益率 离差系数高于 IP0, 但低于并购; 账面回报 的离差系数在三种退出方式中是最低的, 显 示出其低风险的特性。管理层回购退出的投 资金额均值也是最低的, 对资金规模不大的 私募股权机构具有吸引力。

\subsection{2 行业绩效分析}

行业绩效主要从行业的角度分析不同 行业私募股权投资的绩效表现，从而对不同 行业的投资收益进行度量, 分析私募股权投 资的行业选择倾向。本文的行业分类基于国 家统计局的国民经济行业分类标准 (GB/T 4754-2011) 进行分类, 按行业大类共分为 17 种行业, 涵盖行业分类标准中 85\%的行 业。

根据统计结果, 内部收益率最高的是卫 生和社会工作行业 (3.35，335\%), 最低的是 金融业 $(0.52 ， 52 \%)$ ，其中金融业的离散系 数为 0.97 , 比该值高的有 8 个, 低于该值 的有 8 个, 显示出金融业的风险性居中, 但 收益率却非常低, 这从侧面显示出在与业内 已经形成的金融企业竞争时初创金融企业 处于劣势地位。通过进一步分析内部收益率 及其离散系数发现, 从初创企业的角度, 房 地产业并不是一个好的行业选择, 其内部收 益率较低, 离散系数却很高, 这反映出在已 经形成的房地产市场中, 现有的房地产商对 市场的掌控程度较高, 不利于初创企业的发 展。而在诸如建筑、科学研究、教育等领域, 初创企业往往能够获得较好的收益, 且面临 的风险也相对较低。

\section{2.3 公共市场等价分析}

本文通过比较 PME 值探究退出时间对 投资绩效的影响。公共市场等价即 PME 值衡 量的是同等数量的资金投资于非公开交易 市场与投资于公开交易市场两者获利水平 的比较。在剔除无法确定具体日期的案例及 PME' 大于 100 的案例之后, 共剩余 1472 
个案例。

统计可知, 每年的 PME 均值均大于 1 , 最小值出现在 2007 年, 为 2.25 , 意味着私 募投资的收益比将同等数量的资金投资于 公开交易市场多 125\%。这也意味着从均值 的角度, 每年私募投资的收益均高于公开市 场的投资收益。

\section{3 建议}

根据对我国私募股权投资市场绩效的 分析, 在结合我国私募股权投资发展现状的 基础上, 提出以下建议,

（1）完善二级交易市场。制定产权交 易方面的相关举措, 鼓励并购市场的进一步 发展, 促进私募股权投资机构将更多的资源 从 IPO 市场转移到并购市场, 这样既能降 低创业版市场面临的压力, 也能促进资本的 流动, 构建更加合理有效的私募股权投资退 出体系。

（2）加强行业规范。一方面应加强行 业协会在整个行业发展中的监督作用, 加强 信息披露, 促进整个行业向规范化方向发 展。另一方面从投资者的角度, 也应加深对 投资机构的了解, 在投资的过程中做好相关 的尽职调查工作, 切忌因盲目追求高收益而 忽略了投资所面临的风险因素。

(3) 转变投资思路。在投资的退出渠 道方面, 私募股权投资机构应拓宽退出的渠 道选择范围; 在投资的目标选择上, 拓宽投 资的可选择范围, 在不同领域、行业配置资 产，进行分散投资。

（4）完善投资流程。私募股权的投资 大致可以分为四个阶段: 募资、投资、投资 后管理、退出。其中, 最能决定投资绩效的 是投资和退出两个阶段。投资的好坏直接决 定退出方式的选择, 而不同的退出方式也会 带来不同的投资收益。因此, 在投资流程方 面应提升机构的研究能力, 为提升绩效水平 奠定基础。

\section{结束语}

本文首先通过对私募股权投资绩效领 域的相关文献进行总结, 提出本文的研究内 容, 之后在理论层面对私募股权投资的绩效 进行了分析, 并通过搜集私募股权退出案例
对我国私募股权投资的市场绩效进行了实 证分析, 最后在统计分析的基础上得出结论 并提出了促进私募股权投资进一步发展的 建议。但由于数据获取渠道的单一以及个人 能力有限, 仍存在一定的局限和问题: 本文 在分析过程中, 主要从退出方式、行业、时 间的角度对私募股权投资的绩效进行了分 析, 而未对初创企业的发展阶段、私募股权 投资机构的融资轮次等对私募股权投资的 绩效的影响进行深入的研究。因此, 在以后 的研究中, 可考虑利用不同数据库进行交叉 检验, 加入更多的研究变量如私募机构的融 资轮次等对私募股权投资机构的绩效进行 更深入的分析和研究。

\section{致谢}

本文为国家社科基金项目《企业技术跨 越过程中自主创新能力的形成与演化机理 研究》(71272175)的阶段性成果之一。

\section{参考文献}

[1] Paul A. Gompers, Josh Lerner. What drives venture capital fundraising?. NBER Working Paper No.6906. 1999. $1-55$

[2] Brav A. and Gompers, P. A.. Myth or reality? The long-run underperformance of initial public offerings: evidence from venture and nonventure capital-backed companies[J]. Journal of Finance. 1997,52(5): 1791-821

[3] Cochrane J. H. The risk and return of venture capital. Working Paper. 2004.

[4] Jihong Lai. A Study on Private Equity, Enterprise Innovationand the Macro economic Effects $[\mathrm{J}]$. Journal of the Central University of Finance and Economics. 2012,9:42-47

[5] Chao Zheng. Factors Affecting Chinese private equity investment yield [J]. Modern Management Science. 2012,2:44-47

[6] Erxiang Dou. Empirical Research joint investment network investment 
performance impact on private equity [J]. Finance \& Trade Economics.

[7] Yanqiong Mao. Seven Issues on Today $\square \mathrm{s}$ Chinese P rivate Equity Fund. Commercial Research [J]. 2011, 5. 117-121.

[8] Yue Gong. Empirical studies of the impact of private equity funds worth the small board of listed companies [J]. Accounting Monthly. 2012,2:48-51

[9] Shunmu Lin. Invested enterprise performance management and private equity investment fund after International Experience and
2012,10:66-73.

Implications for China [J]. Modern Management Science. 2012,10:58-60

[10] Steven N. Kaplan and Antoinette Schoar. Private equity performance: returns, persistence and capital flows. The Journal of Finance[J]. 2003,(4): 1791-1823

[11] Christian Diller, Christoph Kaserer. What drives private equity returns?fund inflows, skilled GPs, and/or risk? 2005:1-63. 